\title{
How can food extracts consumed in the Mediterranean and East Asia suppress prostate cancer proliferation?
}

\author{
Mu Yao ${ }^{1}$, Chanlu Xie ${ }^{1}$, Maryrose Constantine ${ }^{1}$, Sheng Hua ${ }^{1}$, Brett D. Hambly ${ }^{2}$, Greg Jardine ${ }^{3}$, Paul Sved ${ }^{4}$ \\ and Qihan Dong ${ }^{1,4,5 *}$ \\ ${ }^{1}$ Department of Endocrinology, Central Clinical School and Bosch Institute, Room 394, Blackburn Building, D06, \\ Royal Prince Alfred Hospital, The University of Sydney, Sydney, NSW 2006, Australia \\ ${ }^{2}$ Department of Pathology, The University of Sydney, Sydney, NSW 2006, Australia \\ ${ }^{3}$ Dr Red Nutraceuticals, Mt Nebo, QLD 4520, Australia \\ ${ }^{4}$ Sydney Cancer Centre, Royal Prince Alfred Hospital, Camperdown, NSW 2050, Australia \\ ${ }^{5}$ School of Biomedical and Health Sciences, The University of Western Sydney, Sydney, Australia
}

(Submitted 7 January 2011 - Final revision received 16 September 2011 - Accepted 25 September 2011 - First published online 9 November 2011)

\section{Abstract}

We have developed a blend of food extracts commonly consumed in the Mediterranean and East Asia, named blueberry punch (BBP), with the ultimate aim to formulate a chemoprevention strategy to inhibit prostate cancer progression in men on active surveillance protocol. We demonstrated previously that BBP inhibited prostate cancer cell proliferation in vitro and in vivo. The purpose of this study was to determine the molecular mechanism responsible for the suppression of prostate cancer cell proliferation by BBP. Treatment of lymph node-metastasised prostate cancer cells (LNCaP) and bone-metastasised prostate cancer cells (PC-3 and MDA-PCa-2b) with BBP (up to $0 \cdot 8 \%$ ) for $72 \mathrm{~h}$ increased the percentage of cells at the $G_{0} / G_{1}$ phase and decreased those at the $S$ and $G_{2} / M$ phases. The finding was supported by the reduction in the percentage of Ki-67-positive cells and of DNA synthesis measured by the incorporation of 5-ethynyl-2'-deoxyuridine. Concomitantly, BBP treatment decreased the protein levels of phosphorylated retinoblastoma, cyclin D1 and E, cyclin-dependent kinase (CDK) 4 and 2, and pre-replication complex (CDC6 and MCM7) in LNCaP and PC-3 cells, whereas CDK inhibitor p27 was elevated in these cell lines. In conclusion, BBP exerts its anti-proliferative effect on prostate cancer cells by modulating the expression and phosphorylation of multiple regulatory proteins essential for cell proliferation.

\section{Key words: Proliferation: Cell cycle: Prostate cancer: Blueberry punch}

Active surveillance, rather than immediate aggressive treatment, is emerging as a viable management option for men with prostate cancer. This strategy consists of close monitoring by prostate-specific antigen testing, physical examination, and periodic prostate biopsies aimed to identify the early signs of cancer progression. However, within the next 5 years, $30-40 \%$ of men under active surveillance will experience a rise in prostate-specific antigen or progress to high-grade disease, mandating radical surgery or radiotherapy ${ }^{(1)}$. Thus, developing a chemoprevention strategy to inhibit cancer progression in these otherwise healthy men is of great importance and deserves urgent attention.

We envisage that food-based chemoprevention in combination with active surveillance could benefit these patients. The incidence of clinically important prostate cancer is lower in people living in the Mediterranean and Eastern Asia compared to Western societies ${ }^{(2)}$. However, the incidence of occult prostate cancer was of little variation worldwide ${ }^{(3)}$. It has been proposed that dietary factors are responsible for inhibiting or preventing the progression of occult to clinical prostate cancer ${ }^{(2,4,5)}$. Thus, we have made a concerted effort to develop a blend of food extracts consumed in these regions, named blueberry punch (BBP). We reported recently that $\mathrm{BBP}$ reduced DNA synthesis as measured by thymidine incorporation and topoisomerase levels in prostate cancer cells in vitro and in vivo, respectively. In contrast, an equivalent dose of BBP had little effect on non-cancerous prostate cells $^{(6)}$. The selective effect of BBP on prostate cancer growth could be due to the dependence on phospho-proteinkinase B/AKT, androgen receptor and phospho-cytosolic

Abbreviations: BBP, blueberry punch; CDC6, cell division cycle 6; CDK, cyclin-dependent kinase; EdU, 5-ethynyl-2'-deoxyuridine; LNCaP, lymph nodemetastasised prostate cancer cells; MDA-PCa-2b, bone metastasised androgen receptor positive prostate cells; PC-3, bone metastasised androgen receptor negative prostate cells; pRb, phospho-retinoblastoma.

*Corresponding author: Dr Q. Dong, fax +612 95161273, email qihan.dong@sydney.edu.au 
phospholipase $\mathrm{A}_{2}$ by prostate cancer cells, as all three proteins were reduced after treatment with $\mathrm{BBP}^{(6)}$. In mice, the growth of human prostate cancer cell xenograft was also inhibited with a dose of BBP (10\% of drinking-water) that is applicable in humans ${ }^{(6)}$. The aim of the present study was to determine the molecular mechanism responsible for the suppression of cell proliferation by BBP in human prostate cancer cell lines with different pathological attributes.

\section{Experimental methods}

\section{Blueberry punch preparation}

Fresh olive leaf, grape seed and skin, citrus skin (orange, mandarin, lemon, kaffir lime and Tahitian lime), green tea, turmeric and ginger were juiced individually in a fruit blender. The individual pulps were extracted with 60-95\% ethanol in a ratio of 1:1 in weight for a minimum of 2 weeks. The ethanol-based extraction medium facilitates the extraction of both fat-soluble and water-soluble phytochemicals. A stainless-steel hydraulic press was used to separate the alcoholic extract from pulp. The resulting enriched extracts were blended and subjected to vacuum distillation to remove the alcohol and most of the water. The extracts were then added to blueberry fruit juice with a final concentration of $5 \%$. BBP was manufactured by Dr Red Nutraceuticals Pty Limited (Mt Nebo, QLD, Australia). The major chemicals contained in BBP and their properties were described previously ${ }^{(6)}$.

\section{Cell culture}

Lymph node-metastasised prostate cancer cells (LNCaP; CRL-1740, ATCC) and bone metastasised androgen receptor negative prostate cells (PC-3; CRL-1435; American Type Culture Collection, Manassas, VA, USA) were grown in Roswell Park Memorial Institute (RPMI) 1640 supplemented with 10\% fetal calf serum (Hyclone), penicillin at 100 units/ml and streptomycin at $100 \mu \mathrm{g} / \mathrm{ml}$. Bone metastasised androgen receptor positive prostate cells (MDA-PCa-2b; CRL-2422, ATCC) were grown in F-12K nutrient mixture (Invitrogen) supplemented with 20\% fetal calf serum, penicillin at 100 units $/ \mathrm{ml}$, streptomycin at $100 \mu \mathrm{g} / \mathrm{ml}$, epidermal growth factor $(10 \mathrm{nag} / \mathrm{ml}), 5 \mu \mathrm{m}$-phosphoethanolamine, cholera toxin at $25 \mathrm{ng} / \mathrm{ml}$, hydrocortisone at $0.1 \mathrm{ng} / \mathrm{ml}, 45 \mathrm{~nm}$-selenious acid and insulin at $5 \mu \mathrm{g} / \mathrm{ml}$, as previously described ${ }^{(7)}$. All the cell lines were cultured at $37^{\circ} \mathrm{C}$ in an incubator providing a humidified environment in the presence of $5 \% \mathrm{CO}_{2}$ and $95 \%$ air.

\section{Treatment}

Exponentially growing cells were cultured in six-well plates, T25 flasks or T75 flasks for $3 \mathrm{~d}$ before treatment with BBP. BBP was first diluted to $0.8 \%$ in each culture medium and sterilised by filtration. Further dilutions were made in the corresponding medium to achieve lower concentrations at 0.2 and $0.4 \%$. All cell lines were treated for $72 \mathrm{~h}$ and the untreated cells served as controls.

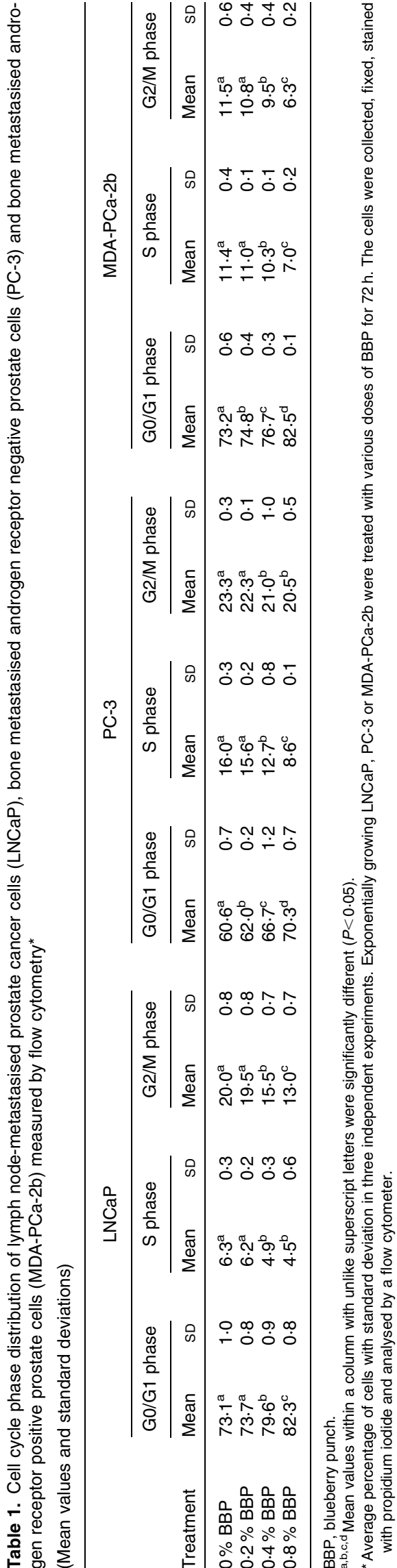




\section{Detection of cellular DNA synthesis}

The cells were treated with BBP in T25 flasks and their DNA synthesis was monitored by the incorporation of 5-ethynyl2'-deoxyuridine (EdU) to newly synthesised DNA with Click-iT ${ }^{\mathrm{TM}}$ EdU Flow Cytometry Assay Kit (C35002; Invitrogen). Following this, $8 \mathrm{~h}$ before completion of treatment, the exhausted medium was removed and the cells were incubated in the fresh medium containing $10 \mu \mathrm{M}$-EdU and BBP at each corresponding concentration. The cells were then trypsinised and fixed in cold $70 \%$ ethanol in PBS at $4{ }^{\circ} \mathrm{C}$ overnight. The fixed cells were then washed with PBS containing 5\% fetal calf serum and the incorporated EdU was labelled with reaction cocktails according to the assay instructions. The labelled cells were analysed by a flow cytometer (BD FACSCalibur; BD Biosciences) equipped with CellQuest Pro software (BD Biosciences) to determine the percentage of cells with incorporated EdU.

\section{Cell cycle analysis}

The cell cycle distribution of the treated cells was determined by flow cytometry. The cells treated in T25 flasks were collected and fixed in cold $70 \%$ ethanol in PBS overnight at $4^{\circ} \mathrm{C}$. After being washed in PBS, the cells were incubated in PBS containing $100 \mu \mathrm{g} / \mathrm{ml}$ RNase and $20 \mu \mathrm{g} / \mathrm{ml}$ propidium iodide at $37^{\circ} \mathrm{C}$ for $60 \mathrm{~min}$. Thereafter, the DNA content was analysed by the flow cytometer.

\section{Immunocytochemistry}

Expression of Ki-67 and phospho-retinoblastoma (pRb) protein at Ser $^{807 / 811}$ was determined by immunostaining. The treated cells in T75 flasks were trypsinised, fixed in $10 \%$ buffered formalin solution overnight at $4{ }^{\circ} \mathrm{C}$, and then processed for paraffin blocks. Sections with $5 \mu \mathrm{m}$ thickness were baked at $60^{\circ} \mathrm{C}$ for $1 \mathrm{~h}$, deparaffinised in xyline, re-hydrated in graded ethanol and distilled water, and subjected to antigen retrieval in Tris-EDTA solution ${ }^{(8)}$. The sections were then blocked with $10 \%$ horse serum and incubated with each primary antibody for $20 \mathrm{~h}$ at $4^{\circ} \mathrm{C}$. The sections were washed with Tris-buffered saline with Tween (TBST) and sequentially labelled with a biotinylated secondary antibody (RA-1000) and Vectastain ABC kit (PK-4000) from Vector Laboratories. The immuno-labelling of indicated antigens was detected with 3,3'-diaminobenzidine (K3468; Dako). The immuno-labelled sections were counterstained with haematoxylin and cover-slipped. The primary antibodies to Ki-67 (RM-9106-S) and pRb at Ser ${ }^{807 / 811}$ (9308) were purchased from Labvision and Cell Signalling Technology, respectively.

\section{Quantification of immunostained cells}

Immunostained sections were scanned by an automated cellular imaging system equipped with automated cellular
(A)

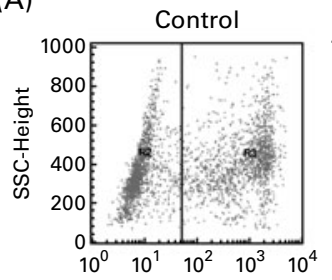

(B)

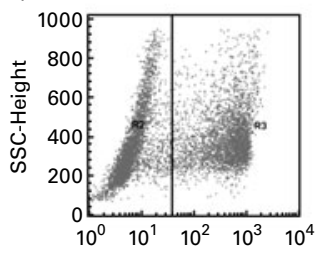

(C)

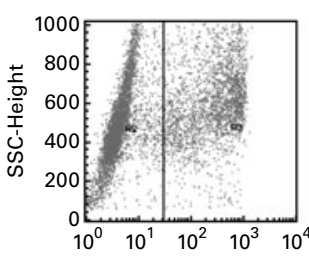

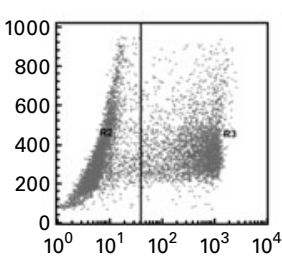

$0.2 \% \mathrm{BBP}$
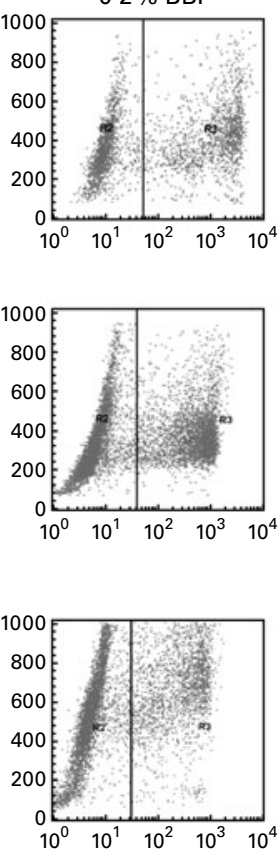
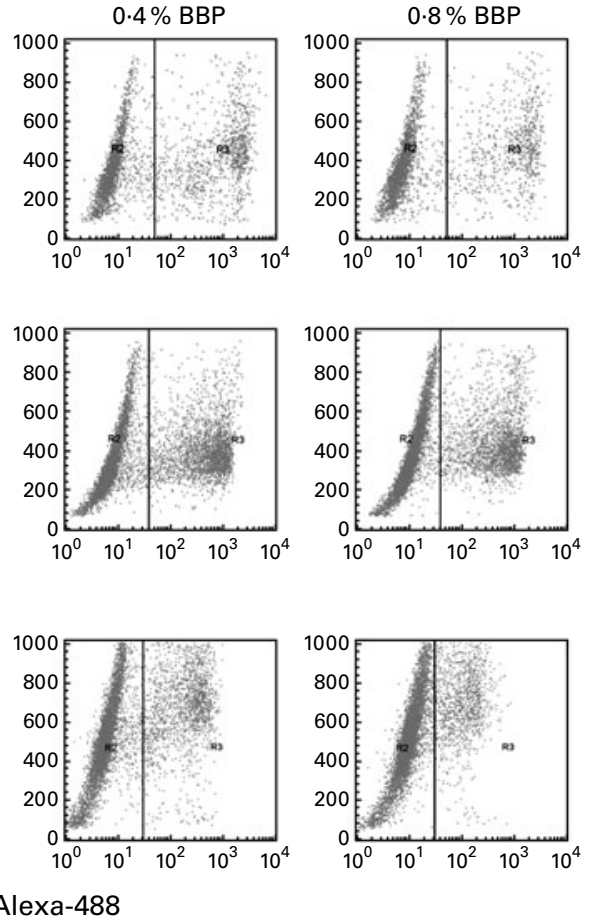
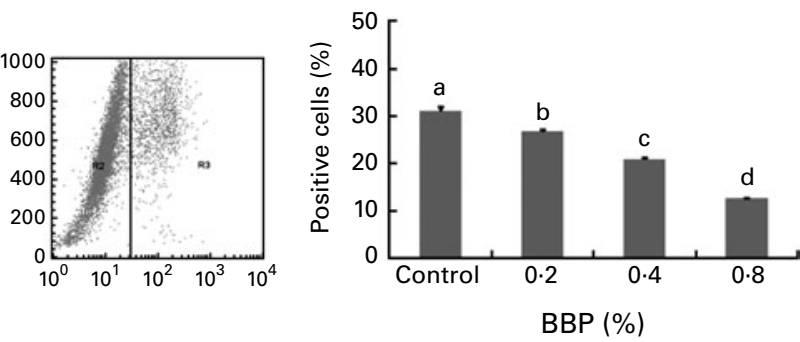

Fig. 1. Effect of blueberry punch (BBP) on DNA synthesis. Exponentially growing (A) lymph node-metastasised prostate cancer cells, LNCap; (B) bone metastasised androgen receptor negative prostate cells, PC-3; and (C) bone metastasised androgen receptor positive prostate cells, MDA-PCa-2b, were incubated with BBP for $72 \mathrm{~h}$, labelled with $10 \mu \mathrm{M}-5$-ethynyl-2'-deoxyuridine for $8 \mathrm{~h}$, and then analysed by flow cytometry as described in the 'Experimental methods' section. Values are means, with standard deviations represented by vertical bars. ${ }^{a, b, c, d}$ Mean values with unlike letters were significantly different by one-way ANOVA followed by multiple-comparison test $(P<0.05)$. 
Table 2. Proliferation of lymph node-metastasised prostate cancer cells (LNCaP), bone metastasised androgen receptor negative prostate cells (PC-3) and bone metastasised androgen receptor positive prostate cells (MDA-PCa-2b) cells measured by Ki-67 staining*

(Mean values and standard deviations)

\begin{tabular}{|c|c|c|c|c|c|c|}
\hline \multirow[b]{2}{*}{ Treatment } & \multicolumn{2}{|c|}{ LNCaP } & \multicolumn{2}{|c|}{ PC-3 } & \multicolumn{2}{|c|}{ MDA-PCa-2b } \\
\hline & Mean & SD & Mean & SD & Mean & SD \\
\hline $0 \%$ BBP & $74.9^{a}$ & 2.9 & $76 \cdot 8^{a}$ & $7 \cdot 3$ & $45 \cdot 7^{\mathrm{a}}$ & \\
\hline $0.2 \%$ BBP & $75 \cdot 9^{\mathrm{a}}$ & 3.0 & $71 \cdot 8^{\mathrm{a}, \mathrm{b}}$ & $2 \cdot 1$ & $35 \cdot 8^{\mathrm{b}}$ & \\
\hline $0.4 \%$ BBP & $59 \cdot 2^{\mathrm{b}}$ & 3.5 & $63.9^{\mathrm{b}}$ & 0.1 & $32.5^{\mathrm{b}}$ & \\
\hline $0.8 \%$ BBP & $24 \cdot 8^{\mathrm{C}}$ & 9.7 & $24 \cdot 8^{\mathrm{C}}$ & $2 \cdot 0$ & $10 \cdot 2^{\mathrm{c}}$ & 1.5 \\
\hline
\end{tabular}

BBP, blueberry punch.

${ }_{a, b, c}$ Mean values within a column with unlike superscript letters were significantly different $(P<0.05)$

${ }^{*}$ Average percentage of Ki-67 positive cells with standard deviation from ten randomly selected fields in three independent experiments. Exponentially growing LNCaP, PC-3 or MDA-PCa-2b were treated with the indicated doses of BBP fo $72 \mathrm{~h}$. Then the cells were harvested, fixed, embedded in paraffin and immunostained for the expression of Ki-67.

imaging system (ACIS) software (ACIS III; Dako) to acquire digital images. Next, two colour thresholds were selected on the images to distinguish between the positively stained (dark brown) and negatively stained (blue) cells. The cells in ten randomly selected fields were then analysed. The percentage of positive cells of each sample was calculated using the following formula:

$$
\text { positive cells } / \text { (positive cells }+ \text { negative cells }) \times 100 \% \text {. }
$$

\section{Immunoblotting}

The cells treated in six-well plates were harvested in lysis buffer (50 mm-Tris-pH 8, $150 \mathrm{mm-NaCl}, 1 \%$ lgepal CA-630, $0.5 \%$ sodium deoxycholate, $0 \cdot 1 \%$ SDS) supplemented with protease inhibitor cocktail (11836145001; Roche) and $50 \mathrm{~mm}$ sodium fluoride. Protein concentration was quantified using a Bio-Rad Protein Assay (Bio-Rad). The proteins in the lysates were separated on SDS-PAGE and transferred onto a nitrocellulose membrane (RPN303E; Amersham Biosciences). The membranes were blocked with $1 \%$ non-fat milk in phosphate-buffered saline-Tween (PBST) for $30 \mathrm{~min}$ and incubated with primary antibody for $1-2 \mathrm{~d}$ at $4^{\circ} \mathrm{C}$. The blots were washed, and incubated with appropriate secondary antibodies conjugated with peroxidase (Sigma-Aldrich) and Precision Strep Tactin-Peroxidase Conjugate (161-0380; Bio-Rad) for $3 \mathrm{~h}$. The immuno-labelling was revealed by SuperSignal West Pico Chemiluminescent Substrate (34078; Thermo Scientific). Immuno-labelled protein bands were captured by a charge-coupled device (CCD) camera built in a gel documentation and analysis system (Syngene). The band intensity on saved digital images was measured using Phoretics 1D Advanced software (Non Linear Dynamics) and the ratio of band intensity between proteins of interest and loading control was calculated. The primary antibodies against Cyclin E (Sc-198), cyclin-dependent kinase (CDK) 2 (Sc-748), CDK4 (Sc-749), cell division cycle (CDC) 6 (Sc9964) and minichromosome maintenance complex (MCM7)
(Sc-22782) were obtained from Santa Cruz Biotechnology; antibodies against Cyclin D1 (C7464) were obtained from Sigma-Aldrich; the antibodies to $\alpha$-tubulin (ab7291) and glyceraldehyde 3-phosphate dehydrogenase (ab8245) were obtained from Abcam.

\section{Statistical analysis}

Statistical comparison was performed using NCSS PASS version 12.0 (NCSS Statistical and Power Analysis Software). The processed data were analysed using one-way ANOVA to determine if a significant change had occurred. Fisher's least significant difference multiple-comparison test (significance: $P<0.05)$ and Kruskal-Wallis multiple-comparison $z$-value test (significance: $z$-value of $>1.96$ ) were applied to determine which doses of BBP had a significant effect.

\section{Results}

\section{Blueberry punch inhibits the proliferation of prostate cancer cells}

To determine the effect of BBP treatment on cell cycle phase distribution, LNCaP, PC-3 and MDA-PCa-2b cells were treated with BBP (up to $0.8 \%$ in corresponding culture medium) for $72 \mathrm{~h}$ and then stained with propidium iodide and analysed by flow cytometry. Treatment with $0 \cdot 2-0 \cdot 4 \%$ BBP blocked cell cycle progression in LNCaP, PC-3 and MDA-PCa-2b cell lines as manifested by an accumulation of cells in the $G_{0} / G_{1}$ phase and a reduction in the percentage of cells in the $S$ or $\mathrm{G}_{2} / \mathrm{M}$ phase (Table 1). A further retardation of cell cycle progression was noted with $0.8 \%$ BBP treatment. To confirm the reduction of cells in the $S$ phase, DNA synthesis was monitored by EdU incorporation using flow cytometry. Treatment with BBP at the concentration of $0 \cdot 2-0 \cdot 4 \%$ decreased DNA synthesis (Fig. 1). A further reduction was noted with $0 \cdot 8 \%$ $\mathrm{BBP}$ in all three cell lines.

To verify the inhibition of cell cycle progression by BBP, the expression levels of $\mathrm{Ki}-67$, a protein associated with cycling

Table 3. Phosphorylation of retinoblastoma protein in lymph nodemetastasised prostate cancer cells (LNCaP), bone metastasised androgen receptor negative prostate cells (PC-3) and bone metastasised androgen receptor positive prostate cells (MDA-PCa-2b) cells*

(Mean values and standard deviations)

\begin{tabular}{|c|c|c|c|c|c|c|}
\hline \multirow[b]{2}{*}{ Treatment } & \multicolumn{2}{|c|}{ LNCaP } & \multicolumn{2}{|c|}{ PC-3 } & \multicolumn{2}{|c|}{ MDA-PCa-2b } \\
\hline & Mean & SD & Mean & SD & Mean & SD \\
\hline $0 \%$ BBP & $27 \cdot 8^{a}$ & 4.9 & $66 \cdot 5^{a}$ & 1.7 & $20 \cdot 7^{a}$ & $2 \cdot 8$ \\
\hline $0.2 \% \mathrm{BBP}$ & $18 \cdot 4^{b}$ & 1.7 & $27 \cdot 6^{\mathrm{b}}$ & $1 \cdot 1$ & $7 \cdot 2^{b}$ & 1.4 \\
\hline $0.4 \%$ BBP & $7 \cdot 9^{c}$ & 1.5 & $11 \cdot 4^{\mathrm{c}}$ & 0.3 & $6 \cdot 7^{\mathrm{b}}$ & $2 \cdot 1$ \\
\hline $0.8 \%$ BBP & $5.9^{c}$ & 1 & $7 \cdot 0^{\mathrm{d}}$ & 0.0 & $3 \cdot 8^{c}$ & 1.5 \\
\hline
\end{tabular}

BBP, blueberry punch.

a,b,c,d Mean values within a column with unlike superscript letters were significantly different $(P<0.05)$.

${ }^{*}$ Average percentage of phospho-retinoblastoma protein-positive cells with standard deviation from ten randomly selected fields in three independent experiments. Exponentially growing LNCaP, PC-3 or MDA-PCa-2b were treated with the indicated doses of BBP for $72 \mathrm{~h}$. Then the cells were harvested, fixed, embedded in paraffin and immunostained for the phospho-retinoblastoma. 
(A)

LNCaP

PC-3

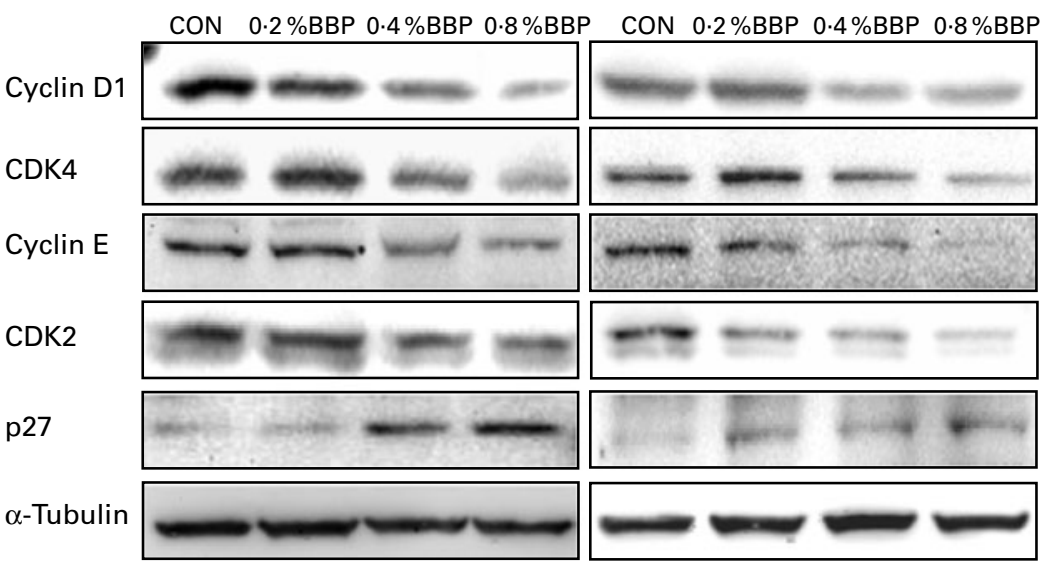

(B)

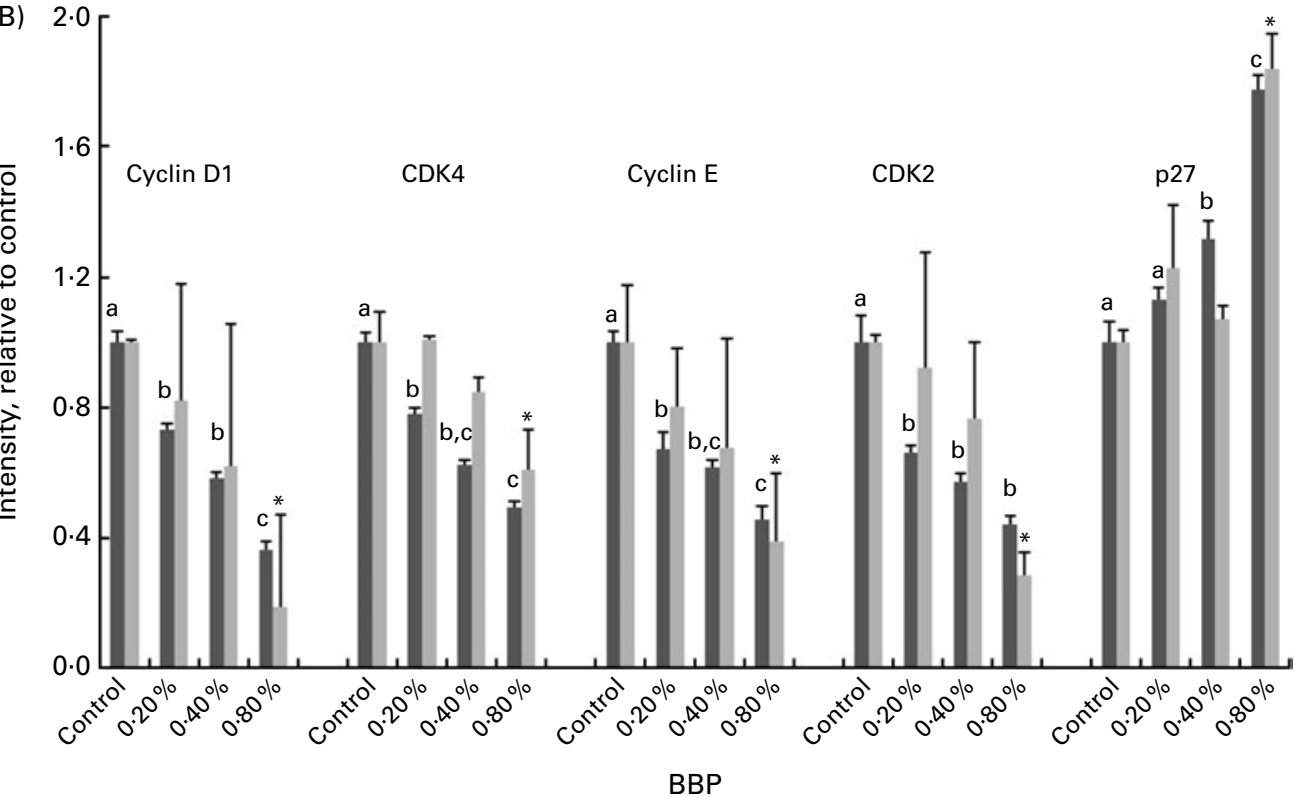

Fig. 2. Effect of blueberry punch (BBP) on the expression of cell cycle regulatory proteins. Exponentially growing lymph node-metastasised prostate cancer cells $(\mathrm{LNCaP})$ and bone metastasised androgen receptor negative prostate cells (PC-3) cells were treated with $0 \% \mathrm{BBP}$ as control (CON) or with the indicated concentrations of BBP for $72 \mathrm{~h}$. (A) Representative immunoblots of cyclin D1, cyclin-dependent kinase (CDK)4, cyclin E, CDK2 and p27. (B) The immuno-labelled proteins on the blots were quantified by densitometry and each protein of interest was normalised by a loading control ( $\alpha$-tubulin or glyceraldehyde 3-phosphate dehydrogenase). The resulting ratio for each dose of BBP was then calibrated by the control $(0 \% \mathrm{BBP})$ that was arbitrarily set as 1 . Values are means, with standard deviations represented by vertical bars of three independent experiments. ${ }^{\mathrm{a}, \mathrm{b}, \mathrm{c}}$ Mean values with unlike letters were significantly different for $\mathrm{LNCaP}(\square ; P<0 \cdot 05)$. ${ }^{*}$ Mean values were significantly different for PC-3 $(\square)$ from those of control $(P<0.05)$.

cells, were determined by immunocytochemistry. Consistently, $0 \cdot 2-0 \cdot 4 \%$ BBP caused a decrease in the percentage of $\mathrm{Ki}-67$ in all three cell lines. There was a further reduction in Ki-67 positivity at $0.8 \%$ BBP (Table 2 and Fig. S1 of the supplementary material, available online at http://www.journals. cambridge.org/bjn). Because phosphorylation on $\mathrm{pRb}$ has been shown to be correlated with cell cycle progression from the $\mathrm{G}_{1}$ to the $\mathrm{S}$ phase ${ }^{(9)}$, the $\mathrm{pRb}$ at $\operatorname{Ser}^{807 / 811}$ was also determined. BBP treatment reduced phosphorylation on $\mathrm{pRb}$ in all treated cell lines to an extent comparable to Ki-67 (Table 3 and Fig. S2 of the supplementary material, available online at http://www.journals.cambridge.org/bjn).
Modulation of cyclins, cyclin-dependent kinase and cyclin-dependent kinase inhibitor by blueberry punch in prostate cancer cells

The aforementioned observations suggest that BBP blocks a transition from the $G_{1}$ to the $S$ phase in treated cells. We therefore determined the levels of cyclin-CDK and their inhibitor P27 involved at $G_{1}-S$ transition in LNCaP and PC-3 cells. BBP treatment decreased the protein levels of cyclin D1/CDK4 and cyclin E/CDK2 in both cell lines (Fig. 2). In LNCaP cells, the observed decreases in the expression of these $G_{1}-S$ transition-regulators are BBP dose-dependent except for CDK2, where a further decrease 
(A)

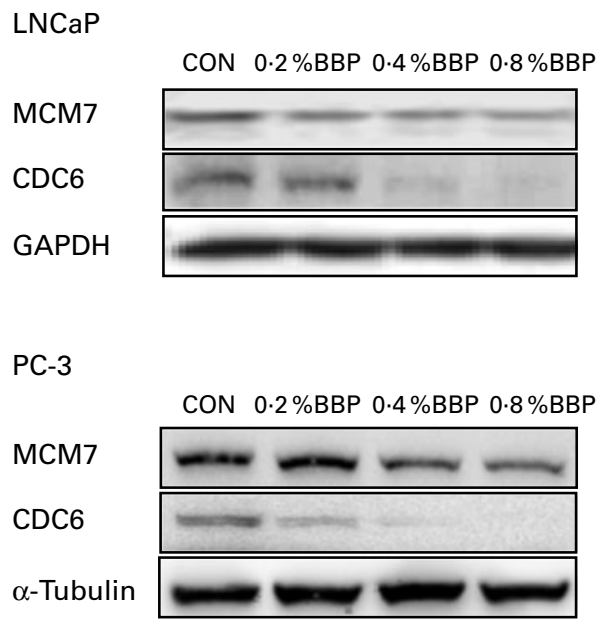

(B)

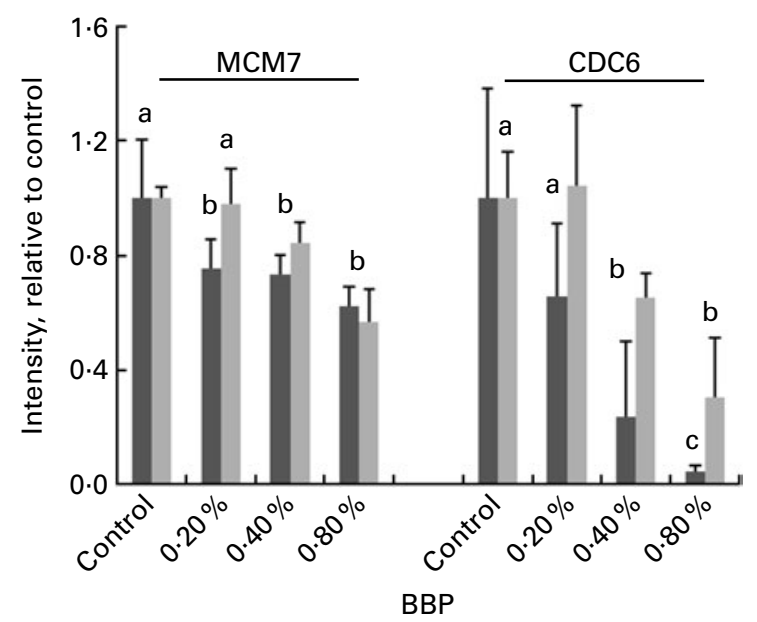

Fig. 3. Effect of blueberry punch (BBP) on the expression of pre-replicative complex proteins. (A) Exponentially growing lymph node-metastasised prostate cancer cells $(\mathrm{LNCaP})$ and bone metastasised androgen receptor negative prostate cells (PC-3) cells were treated with $0 \%$ BBP as control (CON) or with the indicated concentrations of BBP for $72 \mathrm{~h}$ and then analysed for MCM7 and cell division cycle 6 (CDC6) with immunoblot. (B) The immuno-labelled proteins were quantified by densitometry and each protein of interest was normalised by a loading control. The resulting ratio of each BBP concentration was then calibrated by the control $(0 \% \mathrm{BBP})$ that was arbitrarily set to be 1 . Values are means, with standard deviations represented by vertical bars of three independent experiments. ${ }^{a, b, c}$ Mean values with unlike letters were significantly different $(P<0.05)$. $\square, \mathrm{LNCaP} ;, \mathrm{PC}-3$. GAPDH, glyceraldehyde 3-phosphate dehydrogenase.

at $0.8 \%$ BBP did not reach the significance levels. In PC-3 cells, the decrease in cyclin and CDK did not reach statistical significance at $0 \cdot 2-0 \cdot 4 \% \mathrm{BBP}$, but became significant at $0 \cdot 8 \%$ BBP. Concurrently, the treatment elevated the protein levels of $\mathrm{p} 27$ relative to the control in a dose-dependent manner in LNCaP and although there was a trend of increase in p27 in PC-3, a significant increase was noted only at $0.8 \%$ BBP (Fig. 2).

\section{Suppressive effect of blueberry punch on the expression of MCM7 and cell division cycle 6}

The formation of the pre-replication complex is a pre-requisite for DNA replication. MCM7 and CDC6 are the two major components of the pre-replication complex. As BBP inhibited the proliferation in the treated cells, we ascertained whether the MCM7 and CDC6 levels were affected by BBP treatment. As expected, the protein levels of MCM7 and CDC6 in LNCaP and PC-3 cells were reduced by BBP treatment (Fig. 3). In both LNCaP and PC-3 cells, $0 \cdot 2$ or $0 \cdot 4 \%$ BBP caused a decrease in the MCM7 and CDC6 levels. A further decrease at 0.8\% BBP was noted for the CDC6 levels in LNCaP cells. This finding overall was consistent with the observation that BBP restrained DNA synthesis in prostate cancer cells.

\section{Discussion}

In the present study, the anti-proliferative effect of BBP was tested in three prostate cancer cell lines. LNCaP cells were derived from lymph node metastasis and were positive for the androgen receptor ${ }^{(10)}$; while PC-3 cells ${ }^{(10)}$ and MDA$\mathrm{PCa}-2 \mathrm{~b}$ cells ${ }^{(7)}$ were both derived from bone metastasis, and were negative and positive for the androgen receptor, respectively. The present study demonstrates that food extracts commonly consumed in the Mediterranean and East Asia possess a consistent property of anti-proliferation in all three prostate cancer cell lines with different pathological attributes.

Cell cycle analysis revealed that BBP increased the percentage of cancer cells at the $G_{0} / G_{1}$ phase and decreased those at the $S$ and $G_{2} / M$ phases, suggesting a blockade of transition from the $G_{1}$ to the $S$ phase. This is confirmed by a reduction in DNA synthesis by BBP as measured by the incorporation of EdU. A significant decrease in Ki-67 and pRb positive cells after BBP treatment provides further evidence that the proliferation of prostate cancer cells is indeed suppressed. The fact that the treatment reduced the levels of cyclin D1/CDK4 and cyclin E/CDK2, the two complexes essential for regulating the $G_{1}-S$ transition ${ }^{(11-13)}$, and increased the level of $\mathrm{p} 27$, a broad inhibitor of CDK complexes ${ }^{(14)}$, provides the mechanistic evidence for the inhibition of cell cycle progression by BBP. The decrease in the percentage of $\mathrm{pRb}$ positively stained nuclei further supports a decrease in CDK activity by BBP treatment, as $\mathrm{pRb}$ is a substrate of cyclin D1/CDK4 in the early $G_{1}$ and cyclin E/CDK2 in the later $\mathrm{G}_{1}$ phase $^{(14)}$.

DNA replication requires a sequential assembly of licensing factors into pre-replication complex at replication origins, and CDC6 and MCM proteins are the major components of the complex ${ }^{(15,16)}$. Down-regulation of licensing factors suppresses the proliferative capacity and keeps cells in the quiescent state ${ }^{(17)}$. As BBP inhibited the proliferation of the cancer cells, we ascertained whether the CDC6 and MCM7 levels were affected by BBP treatment. The finding regarding the reductions in both $\mathrm{CDC} 6$ and $\mathrm{MCM} 7$ proteins in BBPtreated cells provides further evidence for $\mathrm{BBP}$ to restrain DNA synthesis in prostate cancer cells.

As Ki-67 is only expressed in actively cycling cells including $\mathrm{G}_{1}, \mathrm{~S}, \mathrm{G}_{2}$ and mitosis ${ }^{(18)}$, the decrease in $\mathrm{Ki}-67$ positive cells 
after BBP treatment infers an increase in the number of cells at the $G_{0}$ phase. Thus, we propose that, besides impeding the $\mathrm{G}_{1}-\mathrm{S}$ transition, BBP may cause slippage of cycling cells to a quiescent state or block quiescent cancer cells re-entering the cell cycle. Further studies to ascertain these possibilities will be interesting and of clinical significance.

The results from the present study support and extend our previous finding that BBP possesses anti-proliferative property under both in vitro and in vivo conditions ${ }^{(6)}$. However, it is worth noting that LNCaP and PC-3 cell numbers measured by the MTS assay based on mitochondria activity were reduced by $80 \%$ at $0.6 \% \mathrm{BBP}$ in our previous report ${ }^{(6)}$. The EdU incorporation in the present study was reduced by approximately $50 \%$ at $0.8 \% \mathrm{BBP}$. The relatively smaller reduction in DNA synthesis compared with the decline in cell numbers suggests that BBP may also be cytotoxic to the cancer cells, although this cytotoxic effect is expected to be less than the cytostatic effect.

Considering that alterations in multiple pathways are required for cancer progression, chemoprevention strategy needs to target multiple pathways accordingly. We have shown recently that BBP simultaneously affects the AKT, androgen receptor and cytosolic phospholipase $\mathrm{A}_{2}$ signalling pathways that are essential to the proliferation of prostate cancer cells ${ }^{(6)}$. In the present study, we have provided mechanistic evidence that BBP modulates the levels of key regulators that are involved in the cell cycle progression and the formation of pre-replication complex. Normalisation of aberrant expression of these key regulatory proteins is expected to lead to an inhibition of cancer cell proliferation. Thus, a randomised, double-blind, placebo-controlled clinical trial of BBP should be considered for patients with low-grade and early prostate cancer who are currently under active surveillance.

\section{Acknowledgements}

The present work was supported by Sydney Medical School Foundation (Q. D.) and Dr Red Nutraceuticals (Q. D.). M. Y., C. X., M. C., S. H., B. D. H. and P. S. have no conflicts of interest. Q. D. holds research funds from Dr Red Nutraceuticals and G. J. is an employee of Dr Red Nutraceuticals. M. Y., C. X., M. C., S. H. and G. J. conducted the research; B. D. H. and P. S. analysed the data; M. Y. and Q. D. designed the experiments; and M. Y., C. X., M. C., S. H. and Q. D. prepared the manuscript. The authors thank Masuo Yamaoka (Discovery Unit, Pharmaceutical Research Division, Takeda Pharmaceutical Company) for the MDA-PCa-2b cell line.

\section{References}

1. Dall'Era MA, Konety BR, Cowan JE, et al. (2008) Active surveillance for the management of prostate cancer in a contemporary cohort. Cancer 112, 2664-2670.

2. Itsiopoulos C, Hodge A \& Kaimakamis M (2009) Can the Mediterranean diet prevent prostate cancer? Mol Nutr Food Res 53, 227-239.

3. Ruijter E, van de Kaa C, Miller G, et al. (1999) Molecular genetics and epidemiology of prostate carcinoma. Endocr Rev 20, 22-45.

4. Kouris-Blazos A, Wahlqvist ML, Trichopoulou A, et al. (1996) Health and nutritional status of elderly Greek migrants to Melbourne, Australia. Age Ageing 25, 177-189.

5. Nelson WG, De Marzo AM, DeWeese TL, et al. (2004) The role of inflammation in the pathogenesis of prostate cancer. J Urol 172, S6-S11, (discussion S-2).

6. Singh J, Xie C, Yao M, et al. (2010) Food extracts consumed in Mediterranean countries and East Asia reduce protein concentrations of androgen receptor, phospho-protein kinase B, and phospho-cytosolic phospholipase A(2)alpha in human prostate cancer cells. J Nutr 140, 786-791.

7. Hara T, Nakamura K, Araki H, et al. (2003) Enhanced androgen receptor signaling correlates with the androgen-refractory growth in a newly established MDA PCa 2b-hr human prostate cancer cell subline. Cancer Res 63, 5622-5628.

8. Pileri SA, Roncador G, Ceccarelli C, et al. (1997) Antigen retrieval techniques in immunohistochemistry: comparison of different methods. J Pathol 183, 116-123.

9. Chen PL, Scully P, Shew JY, et al. (1989) Phosphorylation of the retinoblastoma gene product is modulated during the cell cycle and cellular differentiation. Cell 58, 1193-1198.

10. van Bokhoven A, Varella-Garcia M, Korch C, et al. (2003) Molecular characterization of human prostate carcinoma cell lines. Prostate 57, 205-225.

11. Gonzalez MA, Tachibana KE, Laskey RA, et al. (2005) Control of DNA replication and its potential clinical exploitation. Nat Rev Cancer 5, 135-141.

12. Williams GH \& Stoeber K (2007) Cell cycle markers in clinical oncology. Curr Opin Cell Biol 19, 672-679.

13. Braden WA, McClendon AK \& Knudsen ES (2008) Cyclin-dependent kinase $4 / 6$ activity is a critical determinant of pre-replication complex assembly. Oncogene $\mathbf{2 7}$, 7083-7093.

14. Malumbres M \& Barbacid M (2001) To cycle or not to cycle: a critical decision in cancer. Nat Rev Cancer 1, 222-231.

15. Bell SP, Dutta A, Bell SP, et al. (2002) DNA replication in eukaryotic cells. Annu Rev Biochem 71, 333-374.

16. Blow JJ, Hodgson B, Blow JJ, et al. (2002) Replication licensing-defining the proliferative state? Trends Cell Biol 12, $72-78$.

17. Stoeber K, Tlsty TD, Happerfield L, et al. (2001) DNA replication licensing and human cell proliferation. J Cell Sci 114, 2027-2041.

18. Scholzen T \& Gerdes J (2000) The Ki-67 protein: from the known and the unknown. J Cell Physiol 182, 311-322. 